\title{
Mapping the corticoreticular pathway from cortex-wide anterograde axonal
}

\section{tracing in the mouse}

Pierce Boyne, PT, DPT, PhD, NCS; $;^{1}$ Oluwole O. Awosika, MD, MS; ; Yu Luo, PhD ${ }^{3}$

${ }^{1}$ Department of Rehabilitation, Exercise and Nutrition Sciences, College of Allied Health Sciences, University of Cincinnati, Cincinnati, OH, 45267, USA

${ }^{2}$ Department of Neurology and Rehabilitation Medicine, College of Medicine, University of Cincinnati, Cincinnati, OH, 45267, USA

${ }^{3}$ Department of Molecular Genetics, Biochemistry and Microbiology, College of Medicine, University of Cincinnati, Cincinnati, OH, 45267, USA

Key words: motor activity, locomotion, postural balance, pyramidal tracts, extrapyramidal tracts, brain mapping

Grant support: PB is supported by NIH grant R01HD093694. OOA is supported by an American Academy of Neurology Career Development Award. YL is supported by NIH grant R01NS107365.

\section{Corresponding author:}

Pierce Boyne, PT, DPT, PhD, NCS

Health Sciences Building, room 267

3225 Eden Ave.

Cincinnati, OH, 45267-0394

Pierce.Boyne@uc.edu

513-558-7499 (phone); 513-558-7474 (fax) 


\section{ABSTRACT}

The corticoreticular pathway (CRP) has been implicated as an important mediator of

3 motor recovery and rehabilitation after central nervous system damage. However, its origins,

4 trajectory and laterality are not well understood. This study mapped the mouse CRP in

5 comparison with the corticospinal tract (CST). We systematically searched the Allen Mouse

6 Brain Connectivity Atlas (@ 2011 Allen Institute for Brain Science) for experiments that used

7 anterograde tracer injections into the right isocortex in mice. For each eligible experiment

$8(\mathrm{~N}=607), \mathrm{CRP}$ and CST projection strength were quantified by the tracer volume reaching the

9 reticular formation motor nuclei $\left(\mathrm{RF}_{\text {motor }}\right)$ and pyramids respectively. Tracer density in each brain

10 voxel was also correlated with $\mathrm{RF}_{\text {motor }}$ versus pyramids projection strength to explore the relative

11 trajectories of the CRP and CST. We found significant CRP projections originating from the

12 primary and secondary motor cortices, anterior cingulate, primary somatosensory cortex and

13 medial prefrontal cortex. Compared with the CST, the CRP had stronger projections from each

14 region except the primary somatosensory cortex. Ipsilateral projections were stronger than

15 contralateral for both tracts (above the pyramidal decussation), but the CRP projected more

16 bilaterally than the CST. The estimated CRP trajectory was anteromedial to the CST in the

17 internal capsule and dorsal to the CST in the brainstem. Our findings reveal a widespread

18 distribution of CRP origins and confirm strong bilateral CRP projections, theoretically increasing

19 the potential for partial sparing after brain lesions and contralesional compensation after

20 unilateral injury. 


\section{SIGNIFICANCE}

24 The corticoreticular pathway (CRP) provides volitional input to brainstem nuclei that

25 generate walking command signals, facilitate balance and direct limb movements. Upregulation

26 of this pathway appears to be a central mechanism of movement recovery after brain and spinal

27 cord injury, but its anatomy is not well understood. We showed that the mouse CRP originates

28 from widespread parts of the cortex, including non-motor regions, that it projects strongly to both

29 sides of the brainstem, and that its projections are more distributed and bilateral than the

30 corticospinal tract. These findings suggest that the CRP may be particularly resilient to complete

31 disruption.

32 


\section{INTRODUCTION}

The reticulospinal tract is a major motor pathway that delivers the primary input to locomotion-generating circuits in the spinal cord, while also supporting anticipatory postural control and efficient motor synergies in the upper and lower limbs.(Brownstone and Chopek, 2018; Matsuyama et al., 2004; Riddle et al., 2009) The corticoreticular pathway (CRP) provides volitional input to the reticulospinal system via direct and indirect projections from the cerebral cortex to the medial reticular formation motor nuclei $\left(\mathrm{RF}_{\text {motor }}\right)$.(Fisher et al., 2021; Jinnai, 1984; Matsuyama et al., 2004) While the CRP has received much less attention than the more

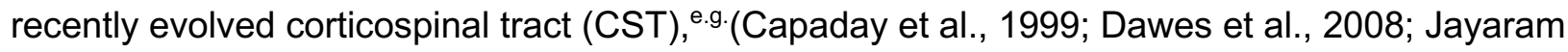

43 et al., 2012; Stinear et al., 2007) accumulating evidence in animal studies (including non-human

44 primates) now indicates that the CRP is likely a critical mediator of motor recovery after central nervous system damage, that also mediates effects of rehabilitation.(Asboth et al., 2018; Darling et al., 2018; Glover and Baker, 2020; Ishida et al., 2019; Takase et al., 2017; Zaaimi et al.,

47 2012; Zaaimi et al., 2018) However, the origins, trajectory and laterality of the CRP are not well understood.

In humans, white matter tracts like the CRP are typically mapped by simulating streamlines through pre-processed diffusion-weighted magnetic resonance images. While this

51 method provides useful non-invasive, in vivo measures, it is limited by insufficient resolution to

52 resolve axonal bundles and inability to determine the direction of neural conduction.(Calamante,

53 2019) Consequently, it is crucial for diffusion tractography to be guided by anatomical

54 knowledge in order to maximize tract coverage and minimize inclusion of false

55 pathways.(Aydogan et al., 2018; Azadbakht et al., 2015; Gutierrez et al., 2020) Unfortunately,

56 this anatomical knowledge is incomplete for the CRP, which limits confidence in prior human

57 CRP mapping.

Most studies in humans and other animals have exclusively mapped CRP projections

59 from the primary and secondary motor areas.(Asboth et al., 2018; Darling et al., 2018; Fisher et 
al., 2021; Fregosi et al., 2017; He and Wu, 1985; Ishida et al., 2019; Jang and Lee, 2019; Jinnai, 1984; Kably and Drew, 1998a; Kably and Drew, 1998b; Lamas et al., 1994; Matsuyama et al., 2004; Pilyavsky, 1975; Schulz et al., 2017; Takase et al., 2017; Yeo et al., 2020) However, the extent of cortical inputs to the CRP has not been well defined, and there have been preliminary indications of potentially important CRP bundles originating from outside the motor cortex.(Keizer et al., 1989; Newman et al., 1989; Rho et al., 1997; Rossi and Brodal, 1956; Shammah-Lagnado et al., 1987) For example, one anterograde axonal tracing study using wheat germ agglutinin-horseradish peroxidase (WGA-HRP) in the rat revealed dense

$68 \mathrm{RF}_{\text {motor }}$ projections from the anterior cingulate cortex $(\mathrm{N}=2)$ and medial prefrontal cortex 69 (N=5).(Newman et al., 1989) Likewise, a classical retrograde tracing experiment using HRP or WGA-HRP injections into $\mathrm{RF}_{\text {motor }}$ in rats $(\mathrm{N}=41)$ found that the strongest CRP projections originated from the anterior cingulate and medial prefrontal cortices.(Shammah-Lagnado et al., 1987) Such projections could be important because they may provide greater opportunity for CRP sparing after a brain lesion, and novel targets for neuromodulation. However, other small studies ( $\mathrm{N}=2-14)$ using anterograde lesion degeneration mapping or classical retrograde tracer injections into the medial reticular formation in the cat or primate have only observed either anterior cingulate(Keizer et al., 1989) or medial prefrontal(Rho et al., 1997; Rossi and Brodal, 1956) CRP origins, or neither.(Berrevoets and Kuypers, 1975; Keizer et al., 1984) Uncertainty about the extent of CRP origins has also been compounded by the limitations of these prior tract tracing experiments testing the breadth of its inputs, which were all

80 from the $20^{\text {th }}$ century. Classical tracers commonly have spread from the injection site, non-

81 specific cell labelling, bidirectional transport (anterograde and retrograde) and trans-synaptic

82 travel, which could have led to false positives.(Saleeba et al., 2019) Likewise, false negatives

83 may have occurred due to insufficient neuronal uptake or transport over long-range CRP

84 axons,(Saleeba et al., 2019) a limited search window or a differing taxonomy of brain regions.

85 Modern tract tracing methods are largely able to overcome these limitations,(Chamberlin et al., 
1998; Oh et al., 2014; Ragan et al., 2012; Saleeba et al., 2019; Wang et al., 2020) but the extent of cortical inputs to the CRP has not been previously assessed systematically with nextgeneration viral tracers or localization procedures.

In addition, the laterality of the CRP also remains incompletely understood. The CRP has been found to project bilaterally to $\mathrm{RF}_{\text {motor, }}$ e.g.(Fisher et al., 2021; Fregosi et al., 2017; Kably and Drew, 1998; Matsuyama and Drew, 1997; Rho et al., 1997) which could have important implications for recovery from unilateral brain lesions.(Brownstone and Chopek, 2018; Jang and Lee, 2019; Takase et al., 2017) However, a paucity of studies have quantified the laterality of these projections,(Fisher et al., 2021; Kably and Drew, 1998; Rho et al., 1997) and those studies have only tested small numbers of neurons originating from restricted sites within the motor cortex,(Fisher et al., 2021; Kably and Drew, 1998) and/or have had relatively small sample sizes (N=4-14).(Fisher et al., 2021; Kably and Drew, 1998; Rho et al., 1997) A broader quantification of CRP lateralization would provide a better understanding of the proportion of contralateral projections available for compensation after unilateral brain injury. knowledge gaps. This open data resource was generated from brain-wide projection mapping, using stereotaxic injections with enhanced green fluorescent protein (EGFP)-expressing adenoassociated viral vectors (rAAV2/1) for anterograde axonal labelling, with negligible retrograde transport.(Chamberlin et al., 1998) Serial two-photon tomography(Ragan et al., 2012) was then followed by spatial registration into a common three-dimensional reference space

107 (CCFv3).(Wang et al., 2020) Labeled pixel volumes were quantified at the injection site and in

108 bilateral atlas regions across the brain, including many specific reticular nuclei.(Oh et al., 2014)

109 This resource has already made major contributions to our understanding of brain

110 organization,(Oh et al., 2014) and it currently includes data from 2,994 anterograde tracer

111 experiments, but it has not been previously used to evaluate the CRP. The current study 
112 leveraged the Allen Connectivity Atlas to determine the location, extent and laterality of cortical

113 inputs to the CRP in the mouse, while also mapping the cortical inputs to the CST for

114 comparison. In addition, we used statistical methods developed for human brain imaging

115 analysis to explore the trajectory of the CRP relative to the CST.

117 METHODS AND MATERIALS

118 Data sources

119 Anterograde tracer data were obtained from the Allen Mouse Brain Connectivity

120 Atlas(Oh et al., 2014) (@ 2011 Allen Institute for Brain Science http://connectivity.brain-

121 map.org/). The experimental procedures that produced this dataset are summarized in the

122 introduction above and were approved by the Institutional Animal Care and Use Committee of

123 the Allen Institute for Brain Science.(Oh et al., 2014) Experiments were performed on adult mice

124 with injections at postnatal day 54-58 and euthanasia 21 days later followed by brain imaging

125 and data processing.(Oh et al., 2014) Further details on the experimental procedures have been

126 described in previous publication.(Oh et al., 2014) We also obtained reference anatomical data

127 and brain region annotation labels from the Allen Mouse Brain Atlas(Sunkin et al., 2006) (@)

1282004 Allen Institute for Brain Science http://atlas.brain-map.org/) in the CCFv3 reference space,

129 from the Scalable Brain Atlas(Bakker et al., 2015)

130 (https://scalablebrainatlas.incf.org/mouse/ABA v3).

132 Data selection \& processing

133 To identify all potentially eligible experiments in the connectivity atlas, we performed a

134 'source search' for those that included injection into the cerebral isocortex using the anterograde

135 EGFP tracer. The following link reproduces the search: http://connectivity.brain-

136 map.org/projection?searchMode=source\&sourceDomain=315\&primaryStructureOnly=true\&trac

137 ers=10\&isi=false. A 'target search' within those results was then performed to find experiments 
138 where tracer was detected in one or more of the reticulospinal motor nuclei involved with limb

139 movement $\left(\mathrm{RF}_{\text {motor; }}\right.$ Table 1; http://connectivity.brain-

140 map.org/projection?searchMode=target\&sourceDomain=315\&primaryStructureOnly=true\&tracer

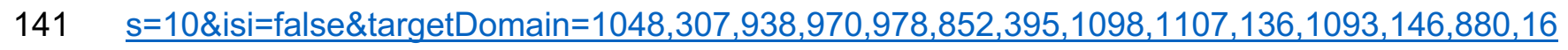

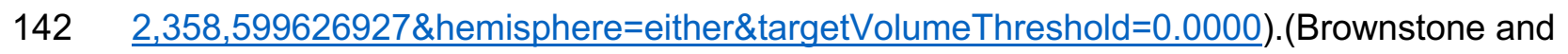

143 Chopek, 2018)

$\ll<$ Insert Table 1 near here $>>>$

One experiment (ID: 249396394) was excluded because the injection site mapped to the

146 superior colliculus (outside of the isocortex). We also restricted our analysis to injection

147 experiments in the right hemisphere, because there was minimal coverage of non-visual regions

148 in the left hemisphere. Since cortical layer 5 is the origin of corticofugal projection pathways like

149 the CRP and CST,(Gerfen et al., 2018) we also excluded experiments that used transgenic

150 mice with limited expression in layer 5, according to the documentation (http://connectivity.brain-

151 map.org/transgenic). This meant that experiments using the following transgenic lines were

152 excluded: Calb1-T2A-dgCre, Calb2-IRES-Cre, Cort-T2A-Cre, Crh-IRES-Cre_BL, Ctgf-T2A-

153 dgCre, Cux2-CreERT2, Cux2-IRES-Cre, Erbb4-T2A-CreERT2, Esr1-2A-Cre, Gad2-IRES-Cre,

154 Grp-Cre_KH288, Htr3a-Cre_NO152, Nos1-CreERT2, Nr5a1-Cre, Ntsr1-Cre_GN220, Oxtr-T2A-

155 Cre, Penk-IRES2-Cre-neo, Pvalb-IRES-Cre, Slc18a2-Cre_OZ14, Syt17-Cre_NO14 and Tac1-

156 IRES2-Cre.

Included experiments used wild-type mice (C57BL/6J) or one of the following transgenic

158 lines: A930038C07Rik-Tg1-Cre, Adcyap1-2A-Cre, Cart-Tg1-Cre, Chrna2-Cre_OE25, Chrnb4-

159 Cre_OL57, Drd3-Cre_KI196, Efr3a-Cre_NO108, Etv1-CreERT2, GIt25d2-Cre_NF107, Gnb4-

160 IRES2-Cre, Gnb4-IRES2-CreERT2, Gng7-Cre_KH71, Gpr26-Cre_KO250, Grm2-Cre_MR90,

161 Htr2a-Cre_KM207, Npr3-IRES2-Cre, Ntng2-IRES2-Cre, Oxtr-Cre_ON66, PIxnd1-Cre_OG1,

162 Pvalb-T2A-CreERT2, Rasgrf2-T2A-dCre, Rbp4-Cre_KL100, Rorb-IRES2-Cre, Rorb-IRES2-Cre-

163 neo, Scnn1a-Tg3-Cre, Sepw1-Cre_NP39, Sim1-Cre_KJ18, Slc17a8-iCre, Slc17a8-IRES2-Cre, 

IRES-Cre.

The projection strength from each cortical injection site to $\mathrm{RF}_{\text {motor }}$ was calculated by dividing the total volume of tracer-labelled pixels in $\mathrm{RF}_{\text {motor }}$ by the injection site volume.(Oh et al., 2014) In a previous study that used this metric for other targets,(Oh et al., 2014) projection strength values $\geq 10^{-3.5}(\sim 0.0003)$ identified false axonal projections (primarily due to small segmentation artifacts) $14.5 \%$ of the time. At a higher threshold of 0.01 , this false positive rate

171 fell to $\sim 0 \%$.(Oh et al., 2014) (extended Fig 7) Thus, we used the 0.01 threshold for descriptive

172 statistics. We also separately calculated the strength of contralateral and ipsilateral projections 173 after repeating the search with left and right sided $\mathrm{RF}_{\text {motor }}$ targets, respectively. To identify CST projections for comparison, we repeated the above procedures with the medullary pyramids as

175 the target structure (http://connectivity.brain-

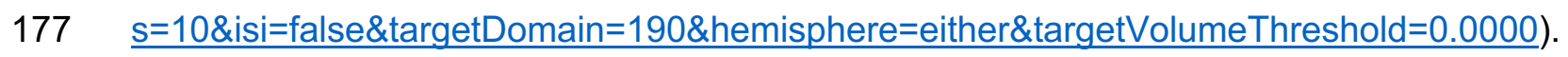

To visualize the results in brain-space, we generated an anatomical surface model of the right hemi-brain without the olfactory bulb in GIFTI format from the $0.025 \mathrm{~mm}$ isotropic resolution CCFv3 atlas (using MATLAB R2017a and Connectome Workbench v.1.4.2). We then mapped the injection coordinates to the nearest surface vertex and labelled a $0.1 \mathrm{~mm}$ radius circle around those coordinates with the projection strength values for that injection. We also

183 mapped the CCFv3 atlas labels for cortical regions of interest (ROls) to the surface model,

184 including the anterior cingulate area, medial prefrontal areas (orbital, prelimbic, infralimbic and

185 frontal pole), secondary motor area, primary motor area and primary somatosensory area.

\section{Data analysis}

188 Projection strength is not normally distributed and has a high frequency of zeros,(Oh et 189 al., 2014) so nonparametric statistics were used for analysis. For each target $\left(\mathrm{RF}_{\text {motor }}\right.$ \& 
pyramids), projection strength was compared between cortical ROls using Kruskal-Wallis ANOVA, followed by Mann-Whitney $U$ pairwise comparisons with false discovery rate (FDR) correction(Benjamini and Hochberg, 1995) across ROIs. For each target, ipsilateral vs. contralateral projection strength was tested using Wilcoxon signed rank tests (paired by injection experiment). We also calculated a projection strength laterality index as: (ipsilateral contralateral) / (ipsilateral + contralateral) * 100, which ranges from -100 (completely contralateral) to 100 (completely ipsilateral), where 0 indicates bilateral symmetry. Between targets, projection strength was compared across all injection experiments, for each cortical ROI, for ipsilateral \& contralateral projections and for the laterality index. These between-target analyses used Wilcoxon signed rank tests (paired by injection experiment) with separate FDR correction across ROls or lateralities. R statistical software(R Development Core Team, 2004) v3.6.0 was used for analysis. The significance threshold was $\mathrm{p}_{\mathrm{FDR}}<0.05$.

\section{Exploratory trajectory comparison analysis} tracts. To address this issue, we performed an exploratory statistical analysis to identify brain voxels more likely to belong to CRP vs. CST projections. For each eligible injection experiment, we downloaded the 3-dimensional projection density image (at $0.05 \mathrm{~mm}$ isotropic resolution), which had been resampled to the CCFv3 atlas space.(Oh et al., 2014) Non-parametric permutation testing was then performed to test how strongly the projection density at each voxel

211 was associated with the CRP vs. CST projection strength across injection experiments, while

212 controlling for the total (whole-brain) projection strength of each experiment. This analysis used

213 FSL randomise(Winkler et al., 2014) and threshold-free cluster enhancement(Smith and

214 Nichols, 2009) with 5,000 permutations and a two-sided significance threshold of $\mathrm{p}_{\mathrm{FDR}}<0.05$.

215 Two-sided significance testing was performed by generating both contrast maps $\left(\mathrm{RF}_{\text {motor }}\right.$ - 
216 pyramids and pyramids $-\mathrm{RF}_{\text {motor }}$ ), taking the minimum $\mathrm{p}$-value in each voxel and multiplying it by

217 two, then running FDR correction. For visualization, results were also projected onto the surface

218 model of the right hemi-brain without the olfactory bulb.

\section{RESULTS}

There were 607 eligible experiments injecting anterograde EGFP tracer into the right isocortex, among which 360 (59.3\%) used male mice and $316(52.1 \%)$ involved injections in the

223 ROls (Fig 1). overall (Fig 2). Within the anterior cingulate, medial prefrontal, secondary motor, primary motor and primary somatosensory ROIs, this proportion was $78.8 \%, 73.9 \%, 80.8 \%, 87.5 \%$ and $64.7 \%$, respectively (Fig 3A). The omnibus Kruskal-Wallis ANOVA revealed significant differences in $\mathrm{RF}_{\text {motor }}$ projection strength among ROls (test statistic $=118.73$, df $=5, \mathrm{p}<2.2 \times 10^{-16}$ ). FDRcorrected pairwise comparisons found that each $\mathrm{ROI}$ had significantly greater $\mathrm{RF}_{\text {motor }}$ projection

231 strength than other (non-ROI) cortical areas, and there were significant differences among ROls

232 (Table 2). The primary motor area had significantly greater $\mathrm{RF}_{\text {motor }}$ projection strength than all

233 other ROls except the secondary motor area, which had significantly greater projection strength

234 than the medial prefrontal and primary somatosensory areas. The anterior cingulate area was

235 not significantly different from the secondary motor, medial prefrontal or primary somatosensory

236 areas. Compared with the pyramids target, projection strength to $\mathrm{RF}_{\text {motor }}$ was significantly

237 greater overall and within all cortical ROls except the primary somatosensory cortex, where the 238 relative projection strengths were equivocal. 
243 (Figs 2 \& 3A, Table 2). The omnibus Kruskal-Wallis ANOVA revealed significant differences in

244 pyramid projection strength among ROls (test statistic $=148.10, \mathrm{df}=5, \mathrm{p}<2.2 \times 10^{-16}$ ). FDR-

245 corrected pairwise comparisons found that the motor and somatosensory areas had significantly

246 greater pyramid projection strength than other (non-ROI) cortical areas, but the anterior

247 cingulate and medial prefrontal areas did not (Table 2). The primary motor area had significantly

248 greater pyramid projection strength than all other ROls except the primary somatosensory area,

249 which had significantly greater projection strength than the anterior cingulate and medial

250 prefrontal areas. The secondary motor area was not significantly different from the primary

251 somatosensory area and also had significantly greater projection strength than the anterior

252 cingulate and medial prefrontal areas. strongly the projection density at each voxel was associated with $R_{\text {motor }}(C R P)$ versus pyramids

262 (CST) projection strength, while controlling for total projection density. A large cluster of voxels 263 with significantly greater $\mathrm{RF}_{\text {motor }}$ association spanned all right cortical ROls and was anterior to

264 the main cluster of greater pyramids association, which included the medial parts of the primary 265 motor and primary somatosensory areas (Fig 4 row 1). In the subcortex, the cluster of greater

$266 \mathrm{RF}_{\text {motor }}$ association followed a trajectory towards the brainstem through the internal capsule that 267 was anterior, ventral and medial to the cluster of greater pyramids association (Fig 4 rows 2-6). 
In the ventral diencephalon and rostral midbrain, the two trajectories crossed and the cluster of greater $\mathrm{RF}_{\text {motor }}$ association became dorsal to the cluster of greater pyramids association. The

270 cluster of greater $\mathrm{RF}_{\text {motor }}$ association then expanded to fill most of the dorsal pons and medulla

271 bilaterally, with multiple areas of apparent decussation, especially in the pons. Meanwhile, the

272 cluster of greater pyramids association followed the compact trajectory of the pyramids in the

273 ventral brainstem and remained primarily ipsilateral before most of the cluster began

274 decussating in the most caudal slices of the medulla.

$$
<<\text { Insert Figure } 4 \text { near here }>>
$$

\section{DISCUSSION}

This study used cortex-wide anterograde axonal tracing data to map the mouse CRP, in comparison with the CST, with an emphasis on cortical inputs and laterality. As expected, the motor cortex provided strong CRP and CST projections. The CRP received its strongest inputs from the primary and secondary motor areas while the CST received its strongest inputs from

282 the primary motor and primary somatosensory areas. Unlike the CST, the CRP also had strong 283 projections originating from the anterior cingulate and medial prefrontal areas. Both tracts had 284 significantly stronger ipsilateral vs. contralateral projections (above the level of the pyramidal 285 decussation), but the CRP was less lateralized, with a greater proportion of bilateral projections.

286 The CRP had greater projection strength than the CST for all ROIs except the primary

287 somatosensory cortex, and for both ipsilateral and contralateral projections.

288 Overall, these results indicate that the CRP is a widely distributed, bilaterally projecting 289 tract in the mouse with more diverse cortical inputs than the CST and thus greater likelihood of 290 partial sparing after brain injury. This is consistent with a recent study that found loss of

291 ipsilesional CST projection strength and upregulation of the ipsilesional CRP, which was

292 correlated with motor recovery after frontoparietal lesions in primates.(Darling et al., 2018) It is 293 also consistent with another recent study showing residual capacity for ipsilesional CRP 
294 upregulation following intracerebral hemorrhage in rats.(Ishida et al., 2019) In addition, the

295 bilateral projection strength of the CRP is reinforced by studies in mice(Takase et al., 2017) and

296 humans(Jang et al., 2013) reporting upregulation of the contralateral CRP after stroke, which

297 was correlated with motor recovery.

298 The current findings appear to diminish long-standing uncertainty from classical tract

299 tracer studies about whether there are CRP projections emanating from the anterior cingulate

300 and medial prefrontal cortices.(Berrevoets and Kuypers, 1975; Keizer et al., 1984; Keizer et al.,

301 1989; Newman et al., 1989; Rho et al., 1997; Rossi and Brodal, 1956) From our large, cortex-

302 wide analysis of next-generation anterograde tracer experiments, it now seems clear that each

303 of these projections is relatively strong, at least in the mouse. Many prior animal studies of the

304 CRP have focused solely on its strongest origins in the motor cortex.(Asboth et al., 2018;

305 Darling et al., 2018; Fisher et al., 2021; Fregosi et al., 2017; He and Wu, 1985; Ishida et al.,

306 2019; Jinnai, 1984; Kably and Drew, 1998; Kably and Drew, 1998; Lamas et al., 1994;

307 Matsuyama et al., 2004; Pilyavsky, 1975; Takase et al., 2017) Thus, future studies are needed

308 to determine the extent to which these additional portions of the CRP might be capable of

309 mediating motor recovery after a brain injury.

310 Our findings also raise pressing questions about the degree to which CRP projections

311 from the anterior cingulate and medial prefrontal cortices may have been preserved in humans.

312 If these projections have been evolutionarily preserved, prior diffusion tractography studies have

313 vastly underestimated the extent of the CRP. Several of these studies have restricted the

314 analysis to projections involving either the precentral gyrus(Lindenberg et al., 2010; Schulz et

315 al., 2017; Zheng and Schlaug, 2015) or secondary motor cortex,(Jang and Seo, 2015; Yeo et

316 al., 2012; Yeo et al., 2020) possibly because initial attempts to map the human CRP without

317 limiting the cortical search window have not found anterior cingulate or medial prefrontal

318 origins.(Soulard et al., 2020; Yeo et al., 2014) However, these studies performed tractography

319 with low-resolution diffusion-weighted imaging and the simple diffusion tensor model, which is 
unable to resolve multiple fiber populations within a voxel and thus highly prone to false negatives (and false positives).(Calamante, 2019) Using a slightly more sophisticated model, Jang and Seo(Jang and Seo, 2014) identified CRP streamlines originating from the dorsal prefrontal cortex, but did not search the anterior cingulate cortex or as far anterior as the medial prefrontal cortex. Thus, it is still possible that CRP bundles originating from the anterior cingulate and medial prefrontal cortices may have persisted in humans. Assessing this possibility with higher resolution diffusion-weighted imaging and next generation modeling methods should be a priority. secondary motor area,(Jang and Seo, 2015; Yeo et al., 2012; Yeo et al., 2020) another important finding was that the primary and secondary motor areas had similar CRP projection strength. Prior tract tracer and invasive neurophysiology studies in animals (including primates) have also consistently found evidence of robust CRP projections originating from the primary motor cortex, including many collateral branches of CST axons.(Asboth et al., 2018; Fisher et

334 al., 2012; Fisher et al., 2021; Fregosi et al., 2017; He and Wu, 1985; Ishida et al., 2019; Jinnai, 335 1984; Kably and Drew, 1998; Kably and Drew, 1998; Lamas et al., 1994; Matsuyama et al., 336 2004) Human CRP tractography studies have identified these primary motor cortex projections 337 too.(Jang and Seo, 2014; Lindenberg et al., 2010; Schulz et al., 2017; Zheng and Schlaug, 338 2015) This indicates that primary motor cortex stimulation or recording likely does not

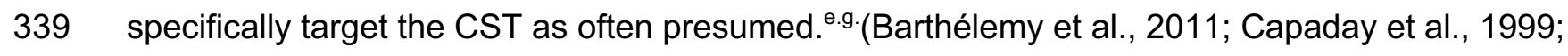
340 Chieffo et al., 2016; Jayaram et al., 2012) It also suggests that projections from the primary 341 motor area should not necessarily be omitted during CRP tractography. Our broad quantification of CRP laterality (Fig 3B \& 3C; Table 2) is consistent with the 343 more focal results from prior studies.(Fisher et al., 2021; Kably and Drew, 1998; Rho et al., 344 1997) For example, Kably and Drew(Kably and Drew, 1998) used microstimulation to measure 345 the laterality of $157 \mathrm{CRP}$ neurons originating from primary or secondary motor areas in the cat 
and found that $49 \%$ were ipsilateral, $35 \%$ were bilateral and $16 \%$ were contralateral. Using

347 retrograde axonal tracing in the cat, Rho et al(Rho et al., 1997) found that the percentage of

348 ipsilateral CRP projections varied from $46.0 \%$ to $72.9 \%$ across different $\mathrm{RF}_{\text {motor }}$ nuclei. In a

349 recent microstimulation study among primates, Fisher et al(Fisher et al., 2021) found bilateral

350 CRP projections to $20 / 36$ (56\%) $R F_{\text {motor }}$ neurons with inputs from the primary motor cortex and

$35130 / 36(83 \%) R_{\text {motor }}$ neurons with inputs from the secondary motor cortex. The current analysis

352 extended these findings by showing the distribution of CRP laterality across cortical injection

353 sites (with high proportions of both ipsilateral and bilateral projection strength) and by

354 quantitatively confirming that the CRP is significantly less lateralized than the CST.

355 In our exploratory voxel-wise analysis testing projection density associations with $\mathrm{RF}_{\text {motor }}$

356 (CRP) versus pyramids (CST) projection strength, the main statistically significant clusters

357 followed paths consistent with plausible CRP trajectories and known CST trajectories (Fig 4). If

358 these statistical results for the CRP are consistent with actual axonal trajectories, it would

359 indicate that the CRP runs anterior, medial and ventral to the CST in the subcortical white

360 matter then moves dorsal to the CST in the ventral diencephalon and rostral midbrain.

361 Interestingly, this is consistent with results from preliminary subcortical CRP mapping with

362 diffusion tractography in humans.(Jang and Seo, 2015) Our results also suggest that the CRP

363 projects bilaterally throughout the dorsal pons and medulla, with multiple decussation points,

364 especially in the pons.

However, other significant association clusters did not match plausible CRP or CST

366 trajectories. The majority of these were in cerebral commissures and contralateral cerebral gray

367 matter regions that were homotopic with ipsilateral clusters and did not project to the brainstem.

368 Thus, we suspect that these clusters were due to confounding from commissural projections

369 that happened to originate near CRP and CST projection neurons, or as collateral branches of

370 CRP or CST axons. When interpreting this analysis, another key consideration is that it was a 
371 contrast between $\mathrm{RF}_{\text {motor }}$ and pyramids projection associations and thus it could not find areas of

372 CRP and CST overlap.

373

374 Limitations

375 An important limitation to this study is that there was incomplete coverage of some 376 cortical subregions, despite the large number of anterograde injection experiments. There was

377 also sparser coverage of the primary motor cortex compared with other ROls. Thus, next-

378 generation retrograde tracer experiments may be able to provide more granular CRP mapping

379 in the future. Another possible issue is that we were only able to include injections in the right

380 isocortex, but there have not been strong indications of interhemispheric CRP differences in

381 prior studies. The projection strength metric used in this analysis does not differentiate tract

382 terminations from continuations, so it could be falsely elevated by sparse fibers passing through

$383 \mathrm{RF}_{\text {motor }}$ or the pyramids without synapsing. Conversely, using transgenic mice and injections at

384 various cortical depths may have falsely lowered projection strength for some experiments,

385 resulting in random measurement error. However, this is unlikely to have caused any systematic

386 error (bias) in the analyses because the CRP and CST results were obtained from the same

387 injections and there is no reason to suspect systematic differences between ROls.

\section{Conclusions}

The mouse CRP bilaterally converged on $\mathrm{RF}_{\text {motor }}$ from large portions of the cortex,

391 including the primary \& secondary motor areas, anterior cingulate, primary somatosensory area

392 and medial prefrontal area, in order of decreasing projection strength. Compared with the CST,

393 the CRP was less lateralized and had stronger projections from all these cortical regions except

394 the primary somatosensory cortex. In the subcortex, the CRP appeared to descend anterior,

395 ventral and medial to the CST before moving dorsal to the CST near the rostral midbrain and

396 projecting bilaterally throughout the dorsal pons and medulla. These findings theoretically 
397 increase the likelihood of partial CRP sparing after brain injury and reinforce the conceptual

398 basis for contralesional CRP compensation after unilateral damage. This foundational

399 information can be used to guide future CRP tractography and projection-specific manipulations.

400 The current study also highlights the value of robust connectomic data generation and

401 sharing(Oh et al., 2014) for enabling ancillary analyses to accelerate scientific progress. 


\section{CONFLICT OF INTEREST STATEMENT}

The authors declare no conflicts of interest.

\section{AUTHOR CONTRIBUTIONS}

Conceptualization, Resources, Software, Formal analysis, Visualization, Writing - original draft: PB. Methodology, Writing - review and editing: PB, OOA, YL.

\section{DATA ACCESSIBILITY STATEMENT}

The data used for this analysis are available from the Allen Mouse Brain Connectivity Atlas (C) 2011 Allen Institute for Brain Science http://connectivity.brain-map.org/). 


\section{REFERENCES}

Asboth L, Friedli L, Beauparlant J, Martinez-Gonzalez C, Anil S, Rey E, Baud L, Pidpruzhnykova G, Anderson MA, Shkorbatova P, Batti L, Pages S, Kreider J, Schneider BL, Barraud Q, Courtine G. 2018. Cortico-reticulo-spinal circuit reorganization enables functional recovery after severe spinal cord contusion. Nat Neurosci 21:576-588.

Aydogan DB, Jacobs R, Dulawa S, Thompson SL, Francois MC, Toga AW, Dong H, Knowles JA, Shi Y. 2018. When tractography meets tracer injections: a systematic study of trends and variation sources of diffusion-based connectivity. Brain Struct Funct 223:2841-2858.

Azadbakht H, Parkes LM, Haroon HA, Augath M, Logothetis NK, de Crespigny A, D'Arceuil HE, Parker GJ. 2015. Validation of High-Resolution Tractography Against In Vivo Tracing in the Macaque Visual Cortex. Cereb Cortex 25:4299-4309.

Bakker R, Tiesinga PHE, Kotter R. 2015. The scalable brain atlas: Instant web-based access to public brain atlases and related content. Neuroinformatics (Totowa, N.J.) 13:353-366.

Barthélemy D, Grey MJ, Nielsen JB, Bouyer L. 2011. Involvement of the corticospinal tract in the control of human gait. Prog Brain Res 192:181.

Benjamini Y, Hochberg Y. 1995. Controlling the False Discovery Rate: A Practical and Powerful Approach to Multiple Testing. J R Statist Soc B 57:289-300.

Berrevoets C, Kuypers H. 1975. Pericruciate cortical neurons projecting to brain stem reticular formation, dorsal column nuclei and spinal cord in the cat. Neurosci Lett 1:257-262.

Brownstone RM, Chopek JW. 2018. Reticulospinal systems for tuning motor commands. Frontiers in Neural Circuits 12:1-10.

Calamante F. 2019. The Seven Deadly Sins of Measuring Brain Structural Connectivity Using Diffusion MRI Streamlines Fibre-Tracking. Diagnostics (Basel) 9:10.3390/diagnostics9030115.

Capaday C, Lavoie BA, Barbeau H, Schneider C, Bonnard M. 1999. Studies on the Corticospinal Control of Human Walking. I. Responses to Focal Transcranial Magnetic Stimulation of the Motor Cortex. J Neurophysiol 81:129-139.

Chamberlin NL, Du B, de Lacalle S, Saper CB. 1998. Recombinant adeno-associated virus vector: use for transgene expression and anterograde tract tracing in the CNS. Brain Res 793:169-175.

Chieffo R, Comi G, Leocani L. 2016. Noninvasive Neuromodulation in Poststroke Gait Disorders: Rationale, Feasibility, and State of the Art. Neurorehabilitation and Neural Repair 30:71-82.

Darling WG, Ge J, Stilwell-Morecraft KS, Rotella DL, Pizzimenti MA, Morecraft RJ. 2018. Hand motor recovery following extensive frontoparietal cortical injury is accompanied by upregulated corticoreticular projections in monkey. The Journal of Neuroscience 38:6323-6339.

Dawes H, Enzinger C, Johansen-Berg H, Bogdanovic M, Guy C, Collett J, Izadi H, Stagg C, Wade D, Matthews PM. 2008. Walking performance and its recovery in chronic stroke in relation to extent of lesion overlap with the descending motor tract. Exp Brain Res 186:325333.

Fisher KM, Zaaimi B, Baker SN. 2012. Reticular formation responses to magnetic brain stimulation of primary motor cortex. J Physiol 590:4045-4060.

Fisher KM, Zaaimi B, Edgley SA, Baker SN. 2021. Extensive Cortical Convergence to Primate Reticulospinal Pathways. J Neurosci 41:1005-1018. 
Fregosi M, Contestabile A, Hamadjida A, Rouiller EM. 2017. Corticobulbar projections from distinct motor cortical areas to the reticular formation in macaque monkeys. Eur $\mathrm{J}$ Neurosci 45:1379-1395.

Gerfen CR, Economo MN, Chandrashekar J. 2018. Long distance projections of cortical pyramidal neurons. J Neurosci Res 96:1467-1475.

Glover IS, Baker SN. 2020. Cortical, Corticospinal, and Reticulospinal Contributions to Strength Training. J Neurosci 40:5820-5832.

Gutierrez CE, Skibbe H, Nakae K, Tsukada H, Lienard J, Watakabe A, Hata J, Reisert M, Woodward A, Yamaguchi Y, Yamamori T, Okano H, Ishii S, Doya K. 2020. Optimization and validation of diffusion MRI-based fiber tracking with neural tracer data as a reference. Sci Rep 10:21285-4.

He X, Wu C. 1985. Connections between pericruciate cortex and the medullary reticulospinal neurons in cat: an electrophysiological study. Experimental Brain Research 61:109-116.

Ishida A, Kobayashi K, Ueda Y, Shimizu T, Tajiri N, Isa T, Hida H. 2019. Dynamic Interaction between Cortico-Brainstem Pathways during Training-Induced Recovery in Stroke Model Rats. The Journal of Neuroscience 39:7306-7320.

Jang SH, Chang CH, Lee J, Kim CS, Seo JP, Yeo SS. 2013. Functional role of the corticoreticular pathway in chronic stroke patients. Stroke 44:1099-1104.

Jang SH, Lee SJ. 2019. Corticoreticular Tract in the Human Brain: A Mini Review. Front Neurol 10:1188.

Jang SH, Seo JP. 2015. The anatomical location of the corticoreticular pathway at the subcortical white matter in the human brain: A diffusion tensor imaging study. Somatosens Mot Res 32:106-109.

Jang SH, Seo JP. 2014. The distribution of the cortical origin of the corticoreticular pathway in the human brain: a diffusion tensor imaging study. Somatosens Mot Res 31:204-208.

Jayaram G, Stagg CJ, Esser P, Kischka U, Stinear J, Johansen-Berg H. 2012. Relationships between functional and structural corticospinal tract integrity and walking post stroke. Clin Neurophysiol 123:2422-2428.

Jinnai K. 1984. Electrophysiological study on the corticoreticular projection neurons of the cat. Brain Res 291:145-149.

Kably B, Drew T. 1998a. Corticoreticular pathways in the cat. I. Projection patterns and collaterization. J Neurophysiol 80:389-405.

Kably B, Drew T. 1998b. Corticoreticular pathways in the cat. II. Discharge activity of neurons in area 4 during voluntary gait modifications. J Neurophysiol 80:406-424.

Keizer K, Keizer K, Kuypers, H. G. J. M., Kuypers, H. G. J. M. 1989. Distribution of corticospinal neurons with collaterals to the lower brain stem reticular formation in monkey (Macaca fascicularis). Experimental Brain Research 74:311-318.

Keizer K, Keizer K, Kuypers, H. G. J. M., Kuypers, H. G. J. M. 1984. Distribution of corticospinal neurons with collaterals to lower brain stem reticular formation in cat. Experimental Brain Research 54:107-120.

Lamas JA, Martinez L, Canedo A. 1994. Pericruciate fibres to the red nucleus and to the medial bulbar reticular formation. Neuroscience 62:115-124. 
Lindenberg R, Renga V, Zhu L, L, Betzler F, Alsop D, Schlaug G. 2010. Structural integrity of corticospinal motor fibers predicts motor impairment in chronic stroke. Neurology 74:280-287.

Matsuyama K, Drew T. 1997. Organization of the projections from the pericruciate cortex to the pontomedullary brainstem of the cat: a study using the anterograde tracer Phaseolus vulgarisleucoagglutinin. J Comp Neurol 389:617-641.

Matsuyama K, Mori F, Nakajima K, Drew T, Aoki M, Mori S. 2004. Locomotor role of the corticoreticular-reticulospinal-spinal interneuronal system. Prog Brain Res 143:239-249.

Newman DB, Hilleary SK, Ginsberg CY. 1989. Nuclear terminations of corticoreticular fiber systems in rats. Brain Behav Evol 34:223-264.

Oh SW, Harris JA, Ng L, Winslow B, Cain N, Mihalas S, Wang Q, Lau C, Kuan L, Henry AM, Mortrud MT, Ouellette B, Nguyen TN, Sorensen SA, Slaughterbeck CR, Wakeman W, Li Y, Feng D, Ho A, Nicholas E, Hirokawa KE, Bohn P, Joines KM, Peng H, Hawrylycz MJ, Phillips JW, Hohmann JG, Wohnoutka P, Gerfen CR, Koch C, Bernard A, Dang C, Jones AR, Zeng H. 2014. A mesoscale connectome of the mouse brain. Nature 508:207-214.

Pilyavsky A. 1975. Characteristics of fast and slow corticobulvar fibre projections to reticulospinal neurones. Brain Res 85:49-52.

R Development Core Team. 2004. R: A Language and Environment for Statistical Computing. R Foundation for Statistical Computing, Vienna, Austria.

Ragan T, Kadiri LR, Venkataraju KU, Bahlmann K, Sutin J, Taranda J, Arganda-Carreras I, Kim Y, Seung HS, Osten P. 2012. Serial two-photon tomography for automated ex vivo mouse brain imaging. Nat Methods 9:255-258.

Rho M, Cabana T, Drew T. 1997. Organization of the projections from the pericruciate cortex to the pontomedullary reticular formation of the cat: A quantitative retrograde tracing study. Journal of Comparative Neurology (1911) 388:228-249.

Riddle CN, Edgley SA, Baker SN. 2009. Direct and indirect connections with upper limb motoneurons from the primate reticulospinal tract. J Neurosci 29:4993-4999.

Rossi G, F , Brodal A. 1956. Corticofugal fibres to the brain-stem reticular formation; an experimental study in the cat. J Anat 90:42-62.

Saleeba C, Dempsey B, Le S, Goodchild A, McMullan S. 2019. A student's guide to neural circuit tracing. Frontiers in Neuroscience 13:897.

Schulz R, Park E, Lee J, Chang WH, Lee A, Kim Y, Hummel FC. 2017. Synergistic but independent: The role of corticospinal and alternate motor fibers for residual motor output after stroke. Neurolmage Clinical 15:118-124.

Shammah-Lagnado SJ, Negra o N, Silva BA, Ricardo JA. 1987. Afferent connections of the nuclei reticularis pontis oralis and caudalis: A horseradish peroxidase study in the rat. Neuroscience 20:961-989.

Smith SM, Nichols TE. 2009. Threshold-free cluster enhancement: Addressing problems of smoothing, threshold dependence and localisation in cluster inference. Neurolmage (Orlando, Fla.) 44:83-98.

Soulard J, Huber C, Baillieul S, Thuriot A, Renard F, Aubert Broche B, Krainik A, Vuillerme N, Jaillard A. 2020. Motor tract integrity predicts walking recovery. Neurology :10.1212/WNL.0000000000008755. 
Stinear CM, Barber PA, Smale PR, Coxon JP, Fleming MK, Byblow WD. 2007. Functional potential in chronic stroke patients depends on corticospinal tract integrity. Brain 130:170-180.

Sunkin SM, Ng LL, Bensinger A, Pak TH, Wood M, Dougherty JG, Fields R, Desaki AL, Diep E, Sarno NR, Bernard A, Chen T, Sutram M, Gates SN, Dolbeare TA, Luong LT, Zhang B, Parry SE, Rockett HR, Smith KA, Jones AR, Crook BE, Stumpf K, Teemer CD, Byrnes EJ, Czaplinska A, Laramee AR, Young RC, Chong J, Youngstrom BL, Smith BI, Howell MP, Sodt AJ, Riley ZL, Smith SC, Ayres M, Feng Yuan X, Wohnoutka PE, Hohmann JG, Ng R, Zwingman TA, Liu Y, Puchalski RB, Faber C, Mosqueda NF, Rowland SA, Overly CC, Mortrud MT, Liang AJ, Wong VY, Dong H, Wolkey CK, Boguski MS, Thompson CL, Duncan BJ, Morgan JJ, Stewart NN, Whitlock RM, Estin LK, Orta GJ, Jeung DP, Larsen KD, Lein ES, Desta T, Kidney JM, Schaffnit K, Lau C, Karr PT, Facer BA, Morgan RJ, Eichele G, Shapovalova NV, Kawal R, Datta S, Glattfelder KJ, Kuan CL, Sivisay T, Thaller C, Chi Chin M, Royall JJ, Lake JH, Visel A, Fliss TP, Fischer SR, Halverson KR, Yaylaoglu MB, Hawrylycz MJ, Ao N, Pearson OC, Varnam LR, Johnson RA, Michaels J, Hart MR, Dee NR, Slaughterbeck CR, Brockway KS, Frensley C, Chen L, Chen L, Dang CN. 2006. Genomewide atlas of gene expression in the adult mouse brain. Nature (London) 445:168-176.

Takase H, Kurihara Y, Yokoyama TA, Kawahara N, Takei K. 2017. LOTUS overexpression accelerates neuronal plasticity after focal brain ischemia in mice. PLoS One 12:e0184258.

Wang Q, Ding SL, Li Y, Royall J, Feng D, Lesnar P, Graddis N, Naeemi M, Facer B, Ho A, Dolbeare T, Blanchard B, Dee N, Wakeman W, Hirokawa KE, Szafer A, Sunkin SM, Oh SW, Bernard A, Phillips JW, Hawrylycz M, Koch C, Zeng H, Harris JA, Ng L. 2020. The Allen Mouse Brain Common Coordinate Framework: A 3D Reference Atlas. Cell 181:936-953.e20.

Winkler AM, Ridgway GR, Webster MA, Smith SM, Nichols TE. 2014. Permutation inference for the general linear model. Neuroimage 92:381-397.

Yeo SS, Chang MC, Kwon YH, Jung YJ, Jang SH. 2012. Corticoreticular pathway in the human brain: diffusion tensor tractography study. Neurosci Lett 508:9-12.

Yeo SS, Jang SH, Park GY, Oh S. 2020. Effects of injuries to descending motor pathways on restoration of gait in patients with pontine hemorrhage. J Stroke Cerebrovasc Dis 29:104857.

Yeo SS, Jang SH, Son SM. 2014. The different maturation of the corticospinal tract and corticoreticular pathway in normal brain development: diffusion tensor imaging study. Front Hum Neurosci 8:573.

Zaaimi B, Edgley SA, Soteropoulos DS, Baker SN. 2012. Changes in descending motor pathway connectivity after corticospinal tract lesion in macaque monkey. Brain 135:22772289.

Zaaimi B, Soteropoulos DS, Fisher KM, Riddle CN, Baker SN. 2018. Classification of Neurons in the Primate Reticular Formation and Changes after Recovery from Pyramidal Tract Lesion. J Neurosci 38:6190-6206.

Zheng X, Schlaug G. 2015. Structural white matter changes in descending motor tracts correlate with improvements in motor impairment after undergoing a treatment course of tDCS and physical therapy. Front Hum Neurosci 9:229. 


\section{TABLES}

\begin{tabular}{|c|c|c|}
\hline $\begin{array}{l}\text { Names in literature } \\
\text { (Brownstone and Chopek, 2018) }\end{array}$ & $\begin{array}{l}\text { Names in connectivity dataset } \\
\text { (Oh et al., 2014) }\end{array}$ & Index \\
\hline \multicolumn{3}{|c|}{ Pontine $\mathrm{RF}_{\text {motor }}$ nuclei } \\
\hline Nucleus reticularis pontis oralis $(\mathrm{PnO})$ & Pontine reticular nucleus (PRNr) & 931 \\
\hline Nucleus reticularis pontis caudalis $(\mathrm{PnC})$ & $\begin{array}{l}\text { Pontine reticular nucleus, caudal } \\
\text { part (PRNc) }\end{array}$ & 913 \\
\hline Ventral tegmental nucleus & Not available & \\
\hline Dorsal tegmental nucleus & Dorsal tegmental nucleus (DTN) & 908 \\
\hline Lateral tegmental nucleus & Not available & \\
\hline Laterodorsal tegmental nucleus (LDT) & $\begin{array}{l}\text { Laterodorsal tegmental nucleus } \\
\text { (LDT) }\end{array}$ & 929 \\
\hline Sublaterodorsal tegmental nucleus (SLDT) & Sublaterodorsal nucleus (SLD) & 934 \\
\hline Ventral nucleus of the medial pontine RF (PnV) & Not available & \\
\hline \multicolumn{3}{|c|}{ Medullary $\mathrm{RF}_{\text {motor }}$ nuclei } \\
\hline $\begin{array}{l}\text { Gigantocellular reticular nucleus (GRN or Gi), } \\
\text { or nucleus reticularis gigantocellularis (NRGc) } \\
\text { or gigantocellular tegmental field (FTG) }\end{array}$ & $\begin{array}{l}\text { Gigantocellular reticular nucleus } \\
\text { (GRN) }\end{array}$ & 975 \\
\hline $\begin{array}{l}\text { Nucleus reticularis magnocellularis (NRMc) or } \\
\text { magnocellular tegmental field (FTM) or pars } \\
\text { alpha and pars ventral of the GRN (GiA \& GiV) }\end{array}$ & $\begin{array}{l}\text { Magnocellular reticular nucleus } \\
\text { (MARN) }\end{array}$ & 984 \\
\hline $\begin{array}{l}\text { Paragigantocellular nucleus dorsal part (DPGi) } \\
\text { and lateral part (LPGi) }\end{array}$ & $\begin{array}{l}\text { Paragigantocellular reticular } \\
\text { nucleus (PGRN), dorsal \& lateral } \\
\text { parts }\end{array}$ & $\begin{array}{l}990- \\
992\end{array}$ \\
\hline Intermediate reticular zone (IRt) & Intermediate reticular nucleus (IRN) & 978 \\
\hline Parvocellular reticular nucleus (PCRt) & $\begin{array}{l}\text { Parvicellular reticular nucleus } \\
\text { (PARN) }\end{array}$ & 988 \\
\hline $\begin{array}{l}\text { Medullary RF dorsal part (MdD) and ventral } \\
\text { part (MdV) }\end{array}$ & $\begin{array}{l}\text { Medullary reticular nucleus } \\
\text { (MDRN), dorsal \& ventral parts }\end{array}$ & $\begin{array}{l}985- \\
987\end{array}$ \\
\hline
\end{tabular}


Table 2. Projection strength to the brainstem targets by cortical injection site and target laterality

\begin{tabular}{|c|c|c|c|c|c|c|c|c|}
\hline & \multirow{2}{*}{$\begin{array}{l}\text { Number } \\
\text { of } \\
\text { injections }\end{array}$} & \multicolumn{3}{|c|}{ Projection strength to $\mathbf{R F}_{\text {motor }}$} & \multicolumn{3}{|c|}{ Projection strength to Pyramids } & \multirow{2}{*}{$\begin{array}{c}\mathrm{RF}_{\text {motor }} \mathrm{VS} . \\
\text { Pyramids } \\
\mathrm{P}_{\mathrm{FDR}}{ }^{*}\end{array}$} \\
\hline & & $\begin{array}{l}\text { Median } \\
(75 \% \text { ile })\end{array}$ & $\begin{array}{l}\mathrm{N}(\%) \\
\geq 0.01\end{array}$ & & $\begin{array}{l}\text { Median } \\
\text { (75\%ile) }\end{array}$ & $\begin{array}{l}\mathrm{N}(\%) \\
\geq 0.01\end{array}$ & & \\
\hline $\begin{array}{l}\text { All eligible experiments, } \\
\text { bilateral targets }\end{array}$ & 607 & $0.02(0.19)$ & $339(55.8)$ & & $0.01(0.06)$ & $303(49.9)$ & & $<0.001$ \\
\hline \multicolumn{4}{|c|}{ By Cortical Injection Site } & $\begin{array}{l}\text { PFDR vs. } \\
\text { Other }^{\dagger}\end{array}$ & & & $\begin{array}{l}\text { PFDR Vs. } \\
\text { Other }\end{array}$ & \\
\hline Anterior cingulate & 52 & $0.10(0.39)$ & $41(78.8)$ & $<0.001^{\mathrm{b}, \mathrm{c}}$ & $0.01(0.03)$ & $22(42.3)$ & $0.43^{\mathrm{c}}$ & $<0.001$ \\
\hline Medial prefrontal & 46 & $0.05(0.19)$ & $34(73.9)$ & $<0.001^{c}$ & $0.00(0.01)$ & $12(26.1)$ & $0.56^{\mathrm{c}}$ & $<0.001$ \\
\hline Secondary motor & 75 & $0.17(0.63)$ & $60(80.0)$ & $<0.001^{\mathrm{a}, \mathrm{b}}$ & $0.06(0.12)$ & $60(80.0)$ & $<0.001^{b}$ & $<0.001$ \\
\hline Primary motor & 24 & $0.28(0.63)$ & $21(87.5)$ & $<0.001^{a}$ & $0.16(0.29)$ & $22(91.7)$ & $<0.001^{a}$ & 0.02 \\
\hline Primary somatosensory & 119 & $0.05(0.29)$ & $77(64.7)$ & $<0.001^{c}$ & $0.08(0.24)$ & $87(73.1)$ & $<0.001^{\mathrm{a}, \mathrm{b}}$ & 0.18 \\
\hline Other cortical & 291 & $0.00(0.03)$ & $106(36.4)$ & reference ${ }^{d}$ & $0.00(0.02)$ & $100(34.4)$ & reference $^{\mathrm{c}}$ & 0.001 \\
\hline \multicolumn{4}{|l|}{ By Target Laterality } & $\begin{array}{c}\text { Pvs. } \\
\text { Ipsilateral }\end{array}$ & & & $\begin{array}{c}\text { P vs. } \\
\text { Ipsilateral }\end{array}$ & \\
\hline Contralateral & 607 & $0.01(0.07)$ & $274(45.1)$ & $<0.001$ & $0.00(0.00)$ & $59(9.7)$ & $<0.001$ & $<0.001$ \\
\hline Ipsilateral & 607 & $0.01(0.10)$ & $312(51.4)$ & reference & $0.01(0.06)$ & $278(45.8)$ & reference & $<0.001$ \\
\hline Laterality Index ${ }^{\ddagger}$ & 607 & $20.2(68.8)$ & $\mathrm{N} / \mathrm{A}$ & $\mathrm{N} / \mathrm{A}$ & $86.8(99.2)$ & $\mathrm{N} / \mathrm{A}$ & $\mathrm{N} / \mathrm{A}$ & $<0.001$ \\
\hline
\end{tabular}

Projection strength $=$ volume of tracer-labelled pixels in the target of $\left(\mathrm{RF}_{\text {motor }}\right.$ or pyramids) divided by injection site volume ${ }^{*}$ P-values from Wilcoxon signed rank tests (paired by injection experiment) with false discovery rate (FDR) correction

${ }^{\dagger} \mathrm{P}$-values from Mann-Whitney U tests with FDR correction

a,b,c,d Rows without a matching letter have pairwise comparisons with $p_{F D R}<0.05$

${ }^{\ddagger}$ Ranges from -100 (completely contralateral) to 100 (completely ipsilateral), where 0 indicates bilateral symmetry 


\section{FIGURES}

Figure 1. Locations of cortical anterograde tracer injections and brainstem targets of interest. Top panel. Each eligible injection experiment $(N=607)$ is marked with a $0.1 \mathrm{~mm}$ diameter black sphere within the translucent brain surface rendering. Cortical regions of interest are outlined on the surface and annotated. In the middle and right images, the left hemi-brain and olfactory bulb have been removed from the surface rendering to better visualize the anterior cingulate and medial prefrontal areas. Bottom panel. From left to right, slices are $x=0.4 \mathrm{~mm}, y=-$ $5.2 \mathrm{~mm}$ and $\mathrm{y}=-7.3 \mathrm{~mm}$. $\mathrm{RF}_{\text {motor, }}$ reticular formation motor nuclei involved with limb movement.

\section{- Tracer Injection Locations}
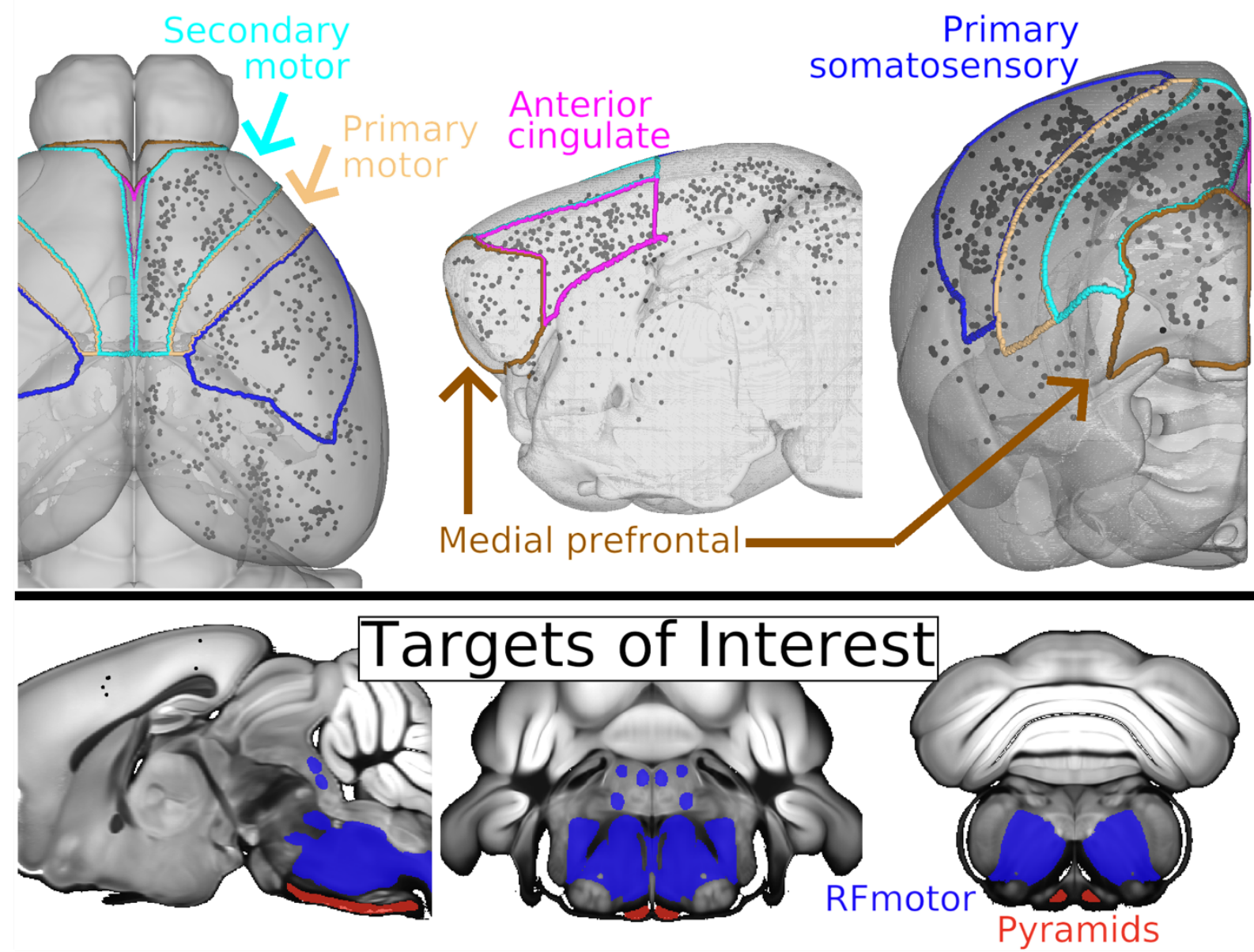
Figure 2. Anterograde tracer projections from cortical injection sites to the reticular formation motor nuclei $\left(\mathbf{R F}_{\text {motor }}\right)$ or medullary pyramids. Projection strength is the volume of tracer-labelled pixels in the target of interest $\left(\mathrm{RF}_{\text {motor }}\right.$ or pyramids) divided by the injection site volume. Results from each eligible injection experiment $(\mathrm{N}=607)$ are mapped onto the nearest surface vertex with a $0.1 \mathrm{~mm}$ radius circle, color-coded with the projection strength from that cortical site to each brainstem target of interest. These results are mapped onto an opaque surface rendering of the right hemi-brain with the olfactory bulb removed for better visualization. Cortical regions of interest are outlined and annotated.

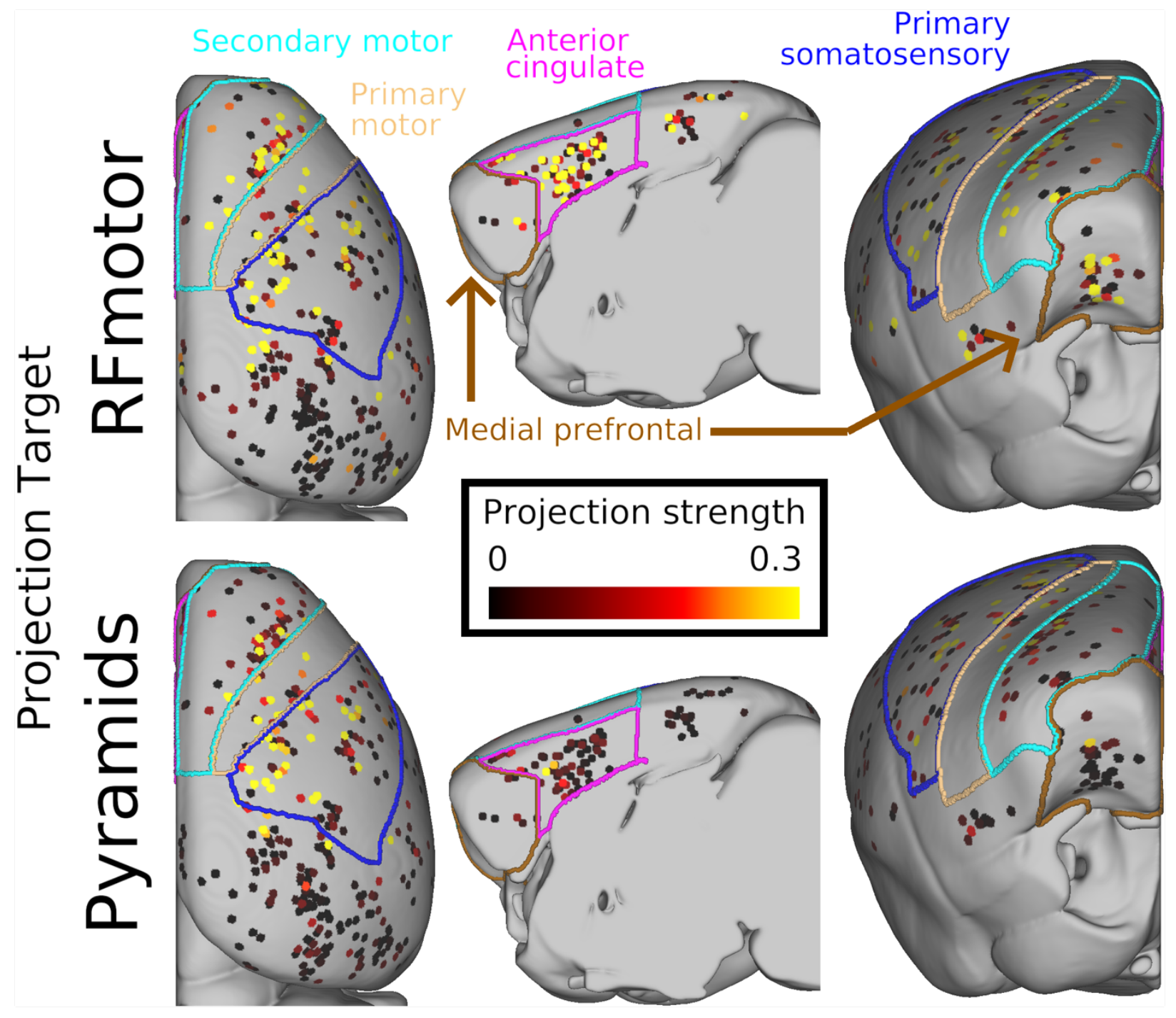


Figure 3. Projection strength to the brainstem targets by cortical injection site and target laterality. Projection strength is the volume of tracer-labelled pixels in the target divided by the injection site volume. Panels A and B. Each eligible injection experiment $(N=607)$ is shown as a + symbol, with projection strength truncated at 1.0 for visualization. Horizontal bars indicate the $75^{\text {th }}$ percentile. Panel C. Distribution of tracer laterality across injection experiments for each target. Vertical bars indicate median laterality indices.
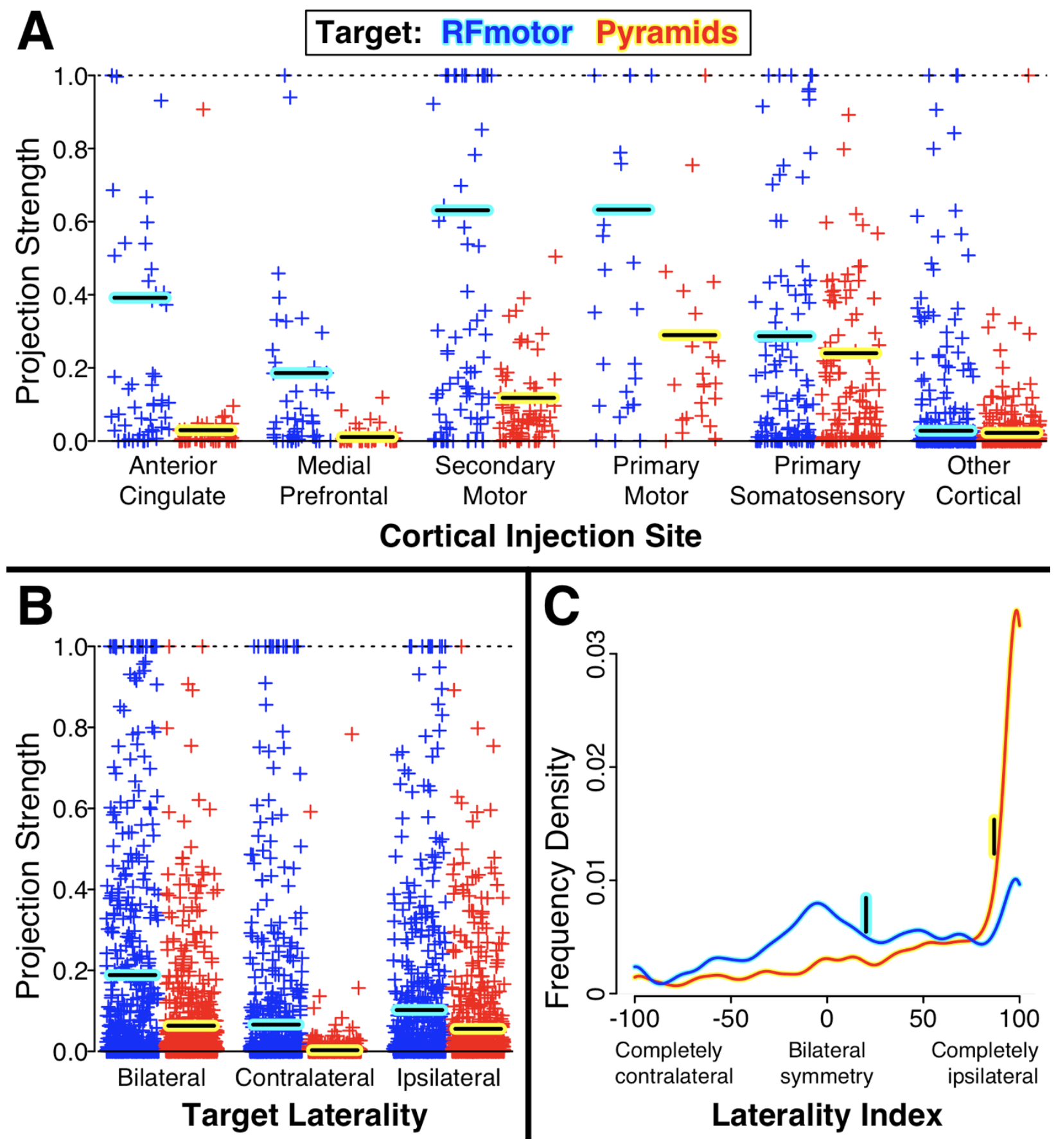

\section{Laterality Index}


Figure 4. Exploratory trajectory analysis of the corticoreticular pathway (CRP) versus the corticospinal tract (CST). Mapped results are T statistics from a general linear model of projection density at each voxel, testing its association with the projection strength to the reticular formation motor nuclei $\left(\mathrm{RF}_{\text {motor }}\right)$ versus the medullary pyramids, while controlling for whole-brain projection strength $(\mathrm{N}=607)$. These results are from non-parametric permutation testing using threshold-free cluster enhancement, and are thresholded at a two-sided, false discovery rate corrected $p<0.05$. Blue $T$ statistics indicate a significantly greater association with $\mathrm{RF}_{\text {motor }}(\mathrm{CRP})$ projection strength, while red/yellow $\mathrm{T}$ statistics indicate a significantly greater association with pyramids (CST) projection strength. The analysis was done in volume space (rows 2-6) and these volumetric images are in neurologic orientation. For visualization, results were also projected onto the surface model of the right hemibrain without the olfactory bulb (row 1). This surface visualization includes black outlines of the cortical regions of interest shown in other figures.

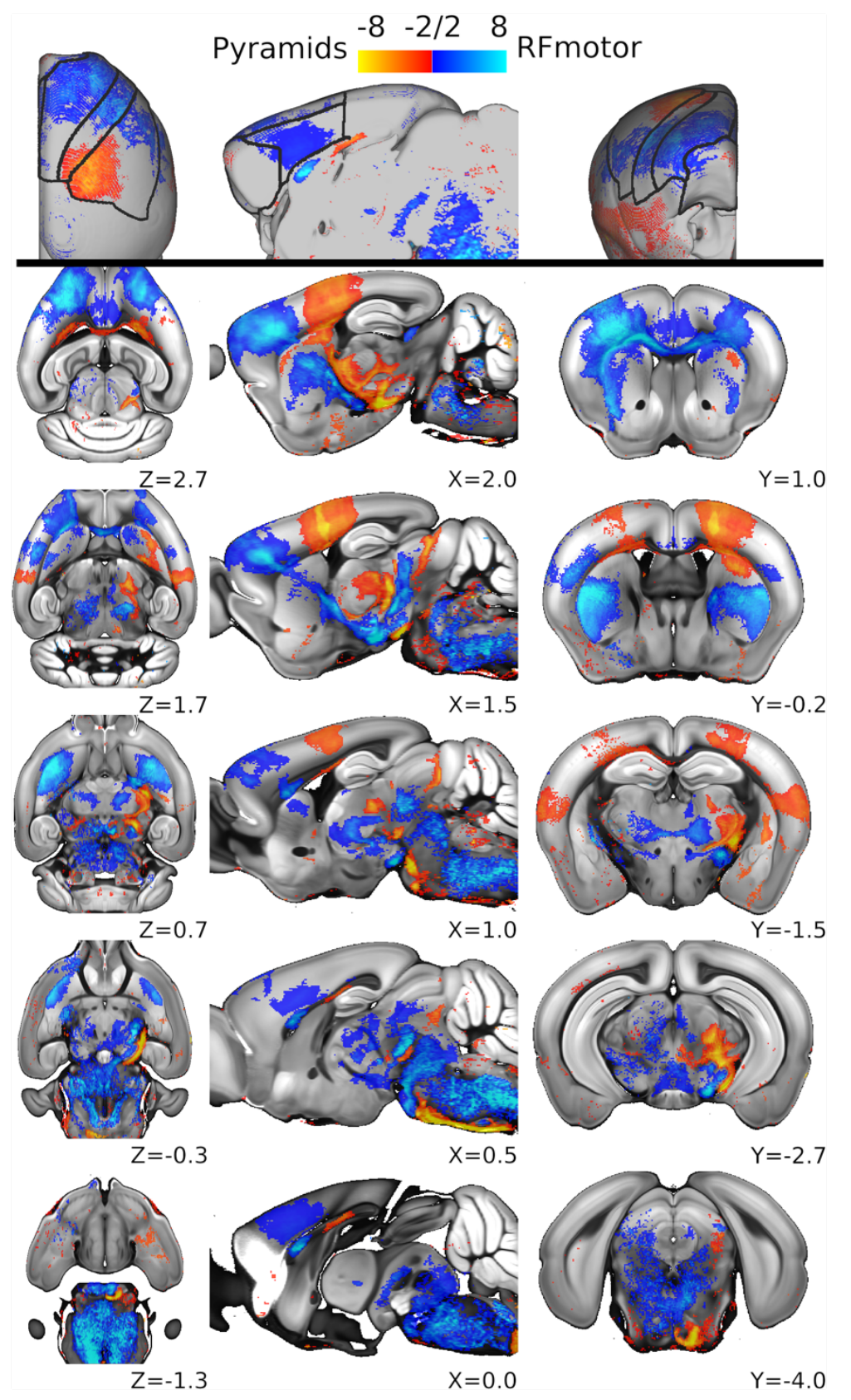

